\title{
Histopathological spectrum of paediatric diffuse intrinsic pontine glioma: diagnostic and therapeutic implications
}

\author{
Pawel Buczkowicz • Ute Bartels • Eric Bouffet • \\ Oren Becher • Cynthia Hawkins
}

Received: 17 March 2014 / Revised: 24 June 2014 / Accepted: 28 June 2014 / Published online: 22 July 2014

(c) The Author(s) 2014. This article is published with open access at Springerlink.com

\begin{abstract}
Diffuse intrinsic pontine glioma (DIPG) is the main cause of brain tumour-related death in children. In the majority of cases diagnosis is based on clinical and MRI findings, resulting in the scarcity of pre-treatment specimens available to study. Our group has developed an autopsy-based protocol to investigate the histologic and biologic spectrum of DIPG. This has also allowed us to investigate the terminal pattern of disease and gain a better understanding of what challenges we are facing in treating DIPG. Here, we review 72 DIPG cases with well documented clinical history and molecular data and describe the pathological features of this disease in relation to clinical and genetic features. Fifty-three of the samples were autopsy material (7 pre-treatment) and 19
\end{abstract}

Electronic supplementary material The online version of this article (doi:10.1007/s00401-014-1319-6) contains supplementary material, which is available to authorized users.

P. Buczkowicz $\cdot$ C. Hawkins $(\square)$

Division of Pathology, The Hospital for Sick Children,

555 University Avenue, Toronto, ON M5G 1X8, Canada

e-mail: cynthia.hawkins@ sickkids.ca

\section{P. Buczkowicz $\cdot$ C. Hawkins}

The Arthur and Sonia Labatt Brain Tumour Research Centre,

The Hospital for Sick Children, Toronto, Canada

P. Buczkowicz $\cdot$ C. Hawkins

Department of Laboratory Medicine and Pathobiology,

Faculty of Medicine, University of Toronto, Toronto, Canada

U. Bartels $\cdot$ E. Bouffet

Division of Haematology and Oncology, The Hospital for Sick

Children, Toronto, Canada

O. Becher

Division of Pediatric Hematology/Oncology, Duke University

Medical Center, Durham, USA were pre-treatment biopsy/surgical specimens. Upon histological review, 62 patients had high-grade astrocytomas (18 WHO grade III and 44 WHO grade IV patients), 8 had WHO grade II astrocytomas, and 2 had features of primitive neuroectodermal tumour (PNET). K27M-H3 mutations were exclusively found in tumours with WHO grade II-IV astrocytoma histology. K27M-H3.1 and ACVRI mutations as well as ALT phenotype were only found in WHO grade III-IV astrocytomas, while PIK3CA mutations and PDGFRA gains/amplifications were found in WHO grade II-IV astrocytomas. Approximately $1 / 3$ of DIPG patients had leptomeningeal spread of their tumour. Further, diffuse invasion of the brainstem, spinal cord and thalamus was common with some cases showing spread as distant as the frontal lobes. These findings suggest that focal radiation may be inadequate for some of these patients. Importantly, we show that clinically classic DIPGs represent a diverse histologic spectrum, including multiple cases which would fit WHO criteria of grade II astrocytoma which nevertheless behave clinically as highgrade astrocytomas and harbour the histone K27M-H3.3 mutation. This suggests that the current WHO astrocytoma grading scheme may not appropriately predict outcome for paediatric brainstem gliomas.

Keywords DIPG $\cdot$ Astrocytoma $\cdot$ Paediatric $\cdot$ Glioma . $\mathrm{H} 3.3 \cdot \mathrm{H} 3 \mathrm{~F} 3 \mathrm{~A} \cdot \mathrm{K} 27 \mathrm{M} \cdot \mathrm{ACVR} 1$

\section{Introduction}

Paediatric brainstem tumours diffusely infiltrative of the pons-diffuse intrinsic pontine gliomas (DIPG) are the most common type of brainstem glioma, accounting for $60-80 \%$ of these tumours [19]. They are currently the 
number one cause of brain tumour-related death in children with a median survival of approximately 10 months and $<10 \% 2$-year survival $[4,13]$. Due to the location of these lesions, surgical intervention is not an option and biopsies have rarely been conducted, with diagnosis based on radiologic features and clinical findings [21]. However, recent biopsy-based studies of DIPG have been safely conducted and may open the door for future biopsy-informed clinical trials [18]. Radiotherapy is the prevailing mode of treatment; however, it is mainly palliative, and clinical trials over the last few decades have not demonstrated a survival benefit of adjuvant chemotherapy [6]. According to a recent review by Jansen et al. [9], there have been 55 clinical trials since 1984, including 26 between 2007 and 2012, with no improved survival or prognosis when compared to radiation therapy alone.

Several studies into the underlying biology of DIPG over the last few years have demonstrated genetic signatures commonly present in subsets of patients $[5,10,16$, 17, 24, 26]. TP53 represents one of the most commonly mutated genes described in DIPG, present in $40-77 \%$ of cases $[5,10]$. Our group and others have demonstrated a high-frequency heterozygous mutation of histone H3.3 in DIPG at residue K27 [10, 24]. The mutation, resulting in replacement of a lysine with methionine (K27M), is present in approximately $70 \%$ of DIPGs versus $21 \%$ of, largely midline thalamic, supratentorial GBMs [10, 22]. $\mathrm{K} 27$ is an important residue that can be either acetylated, methylated or ribosylated on all histone $\mathrm{H} 3$ variants [2, $14,15,20]$. Although the biological consequence of this mutation is still under investigation, given the high frequency of this mutation involving a functionally important, conserved residue, it is likely relevant for tumourigenesis. The roles of K27M-H3.3 in dysregulation of PRC2 complex member EZH2 and global decrease in H3K27me3 have been documented [1, 11]. Moreover, the mutation confers a worse overall survival compared to H3.3 wild-type patients and there are specific copy number alterations associated with the mutation including PDGFRA and PVTI/MYC locus gains and amplification, which are not seen in the wild-type patients [10]. While our understanding of some of the genetic underpinnings of DIPG has improved over the last few years, the assumption has been that these represent brainstem GBMs and that low-grade histology seen in biopsy specimens represents sampling bias. Here, we describe the histopathological features of 72 DIPGs, the largest DIPG histologic series reviewed to date, and correlate it with clinical data as well as recurrent copy number alteration and mutation status of genes frequently affected in DIPG. These data will be crucial for informing future biopsy-based clinical trials.

\section{Patients and methods}

Patient cohort

Seventy-two infants and children diagnosed with DIPG between 1984 and 2012 were included in the study. Fortyone were diagnosed and treated at the Hospital for Sick Children and 31 cases were referred from outside. The cooperating pathologists and institutions are listed in acknowledgements. Clinical presentation was consistent with DIPG including history of one or more of progressive headache, vomiting, diplopia, unsteady gait, slurred speech, papilledema, cranial nerve palsies and signs of ataxia. Imaging characteristics were also in keeping with a diffuse pontine tumour, including a contrasting lesion on MRI with diffuse involvement of at least $50 \%$ of the pons required for DIPG diagnosis. Thirty-five of the patients were female and 37 were male. The mean age of diagnosis for our cohort was 6.87 years (median 6.47 years; range 0 days to 15.35 years). More than $80 \%$ of patients were under the age of 10 at the time of diagnosis. The median survival was 0.83 years.

Chemotherapy, if any, varied based on protocols available at the time of presentation or progression and included temozolomide (Temodol), nimotuzumab (TheraCIM), tipifarnib (Zarnestra), retinoic acid, bevacizumab (Avastin), temsirolimus (Torisel) or cituxumab (IMC-A12).

Radiotherapy data were available for 59 patients. Of these, all but 6 patients received focal radiation. Forty-eight patients received a dose of 54 Gy over 30 fractions. One patient received 25 Gy over 5 days. Three patients received $56 \mathrm{~Gy}$ and one patient received $59 \mathrm{~Gy}$ of radiation. This same patient was re-irradiated. All clinical and molecular features of our DIPG cohort can be found in Table S1.

\section{Histology and immunohistochemistry}

All available surgical and autopsy materials were examined by routine haematoxylin and eosin (H\&E) staining and as indicated, by immunohistochemical staining (IHC) for selected proteins (including MIB1, GFAP, p53, MAP2, NeuN, and nestin) using standard automated immunoperoxidase detection. $5 \mu \mathrm{m}$ sections were cut from paraffin blocks and mounted on positively charged slides. Immunodetection was performed with the automated Benchmark XT stainer (Ventana, Tucson, AZ) using the Ultraview Universal DAB Detection kit (Ventana). Slides were counterstained with haematoxylin II kit (Ventana). All cases were assigned a diagnosis and grade according to WHO criteria. For 44 DIPG patients the entire brain or partial brain specimen with detailed autopsy report was available for examination, allowing extensive histological review of 
tumour spread and statistical testing related to tumour dissemination. For the cases where entire brain was available, 15-20 blocks were taken including approximately ten sections from the brainstem (medulla, pons and midbrain) as well as sections from the thalamus, basal ganglia, occipital lobe, temporal lobe, frontal lobe and deep white matter. Sections were examined and evaluated for extent of disease and presence of leptomeningeal and/or intraventricular dissemination. An additional nine autopsy cases referred from collaborating pathologists and institutions had insufficient autopsy material to evaluate leptomeningeal dissemination or distant tumour spread, but were evaluable for WHO diagnosis and grade and had material available for molecular diagnosis. A further 19 cases had available surgical pretreatment material and were only assessed for tumour grade and molecular analysis when sufficient biological material was available. Where applicable, pathological reports from referring pathologists were reviewed to corroborate histopathological diagnosis of reviewed slides.

\section{Molecular analysis}

Fifty-one DIPG patients had sufficient biological material to perform targeted sequencing of $H 3 F 3 A$, HISTIH $3 B / C$, PIK3CA, TP53 and ACVR1 using Fluidigm array and ion torrent chips (Life Technologies). An additional 15 samples were sequenced for coding regions of $H 3 F 3 A$ and $H I S T 1 H 3 B / C$ using Sanger fluorescence sequencing. The histone $\mathrm{H} 3$ and TP53 mutational status of a subset of these patients has previously been reported [10]. ACVR1 mutations in a subset of these patients were previously described [3]. Alternative lengthening of telomeres was assessed as previously described by either terminal restriction fragment assay (TRF) [8] or C-circles [7].

\section{Statistical analysis}

Where applicable, statistical analysis was performed using SPSS v21 (IBM Corporation) or GraphPad Prism 5 software. Overall survival was estimated using the KaplanMeier method and univariate assessments of Kaplan-Meier plots were tested using log rank. Unpaired two-tailed Student's $t$ test was used for statistical testing of continuous scale data. $p$ values $\leq 0.05$ were considered to be significant. Multivariate Cox proportional hazard models and significance testing based on the Wald test $(\alpha=0.05)$ were used for multivariate analysis. Statistical testing of leptomeningeal dissemination, histone $\mathrm{H} 3$ mutations and histology were employed only for samples with sufficient clinical and molecular data. Statistics on leptomeningeal dissemination was not reported for biopsy patients as tissue specimens were insufficient to determine the presence or absence of leptomeningeal disease.

\section{Results}

DIPG represents a histologic spectrum with grade not predictive of survival

Upon pathological review, 8 (11\%) samples were found to be diffuse astrocytoma WHO grade II (LGA), 18 (25\%) were anaplastic astrocytoma (AA, WHO grade III), 44 $(61 \%)$ were glioblastoma (GBM, WHO grade IV), and $2(3 \%)$ were primitive neuroectodermal tumours (PNET, WHO grade IV). The PNETs were more sharply demarcated from adjacent brain than the glial tumours, had no vascular endothelial proliferation or pseudopalisading necrosis and were immunonegative for GFAP (Fig. 1a). For 17 of the GBM cases we reviewed the histology at multiple levels of the brainstem as well as the thalamus, frontal cortex and metastatic disease and recorded the histologic grade of the tumour at that level (Table 1). Interestingly, this analysis showed that in $7(40 \%)$ cases the GBM histology (vascular endothelial proliferation and necrosis) was limited to the pons while other levels showed WHO grade II or III histology. In no case was GBM histology present at all levels of the brain.

We had only one DIPG patient for whom biopsy and post-mortem material was available. This showed AA histology on biopsy and GBM histology on autopsy. However, as described above it is quite possible to target lower-grade areas on biopsy, thus it is unclear if this represents true progression of disease. Future studies with larger series of matched biopsy/autopsy specimens would need to be conducted in order to determine if anaplastic progression occurs in DIPG between diagnosis and autopsy.

There was no significant difference in survival based on histology (Fig. 2a, $p=0.407$ ). Median survival for patients with diffuse astrocytoma grade II, anaplastic astrocytoma and GBM was $0.88,0.83$ and 0.85 years, respectively. However, the association of age of diagnosis and tumour grade was significant $(p=0.040)$. The age of diagnosis for the glial tumours increased with tumour grade, as LGA, AA, and GBM had mean age of diagnosis at ages 4.19, 6.67 and 7.61 years, respectively. This same trend was observed when biopsy samples and autopsy samples were analysed individually (Table S2).

\section{DIPGs exhibit leptomeningeal spread and distant spread}

For 44 patients sufficient tissue was available to assess extent of spread and the presence of disseminated disease. Seventeen of 44 patients $(38.6 \%$ ) had leptomeningeal spread at autopsy (for example, see Fig. 1b). Further, $11 / 44(25 \%)$ patients had tumour cells present beyond the brainstem (spinal cord and/or thalamic involvement; for example, see Fig. 1c), including four patients with tumour 

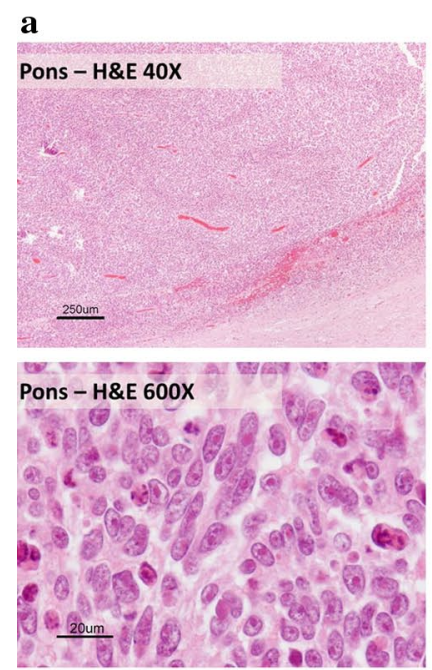

c
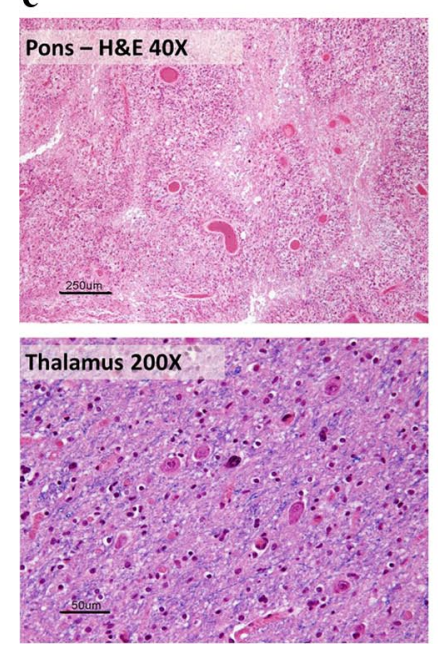
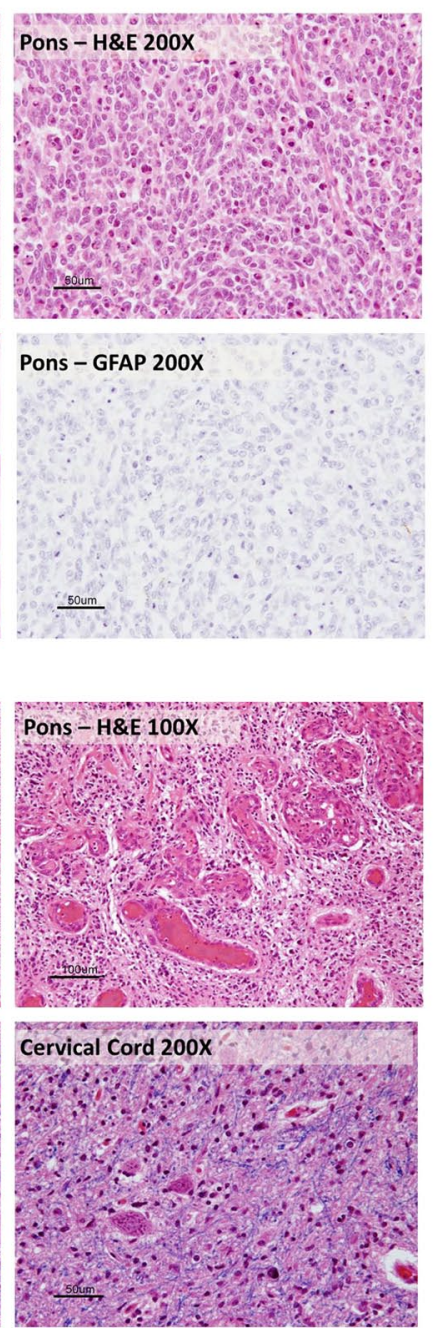

Fig. 1 Histologic spectrum of DIPG. a PNET, WT-H3: the tumour was not directly invasive and sharply demarcated from adjacent brain (upper right panel). On $\mathrm{H} \& \mathrm{E}$ the tumour cells have little cytoplasm with anaplastic nuclei and prominent nucleoli. There was no vascular endothelial proliferation and no pseudopalisading necrosis typical of high-grade astrocytomas. On IHC the tumour cells were negative for GFAP (lower right panel). b LGA, K27M-H3.3: On H\&E this was an infiltrative astrocytic tumour with no high-grade features (WHO grade II). There was no vascular endothelial proliferation, necrosis and very few mitoses (upper left panel). Less than $1 \%$ of tumour cells were positive for MIB1/Ki-67 (upper right panel).

cells diffusely infiltrating as far rostrally as the frontal lobe. There was no statistical correlation between presence of leptomeningeal dissemination and histologic grade. While it did not reach statistical significance, our data suggest that overall survival for patients with leptomeningeal spread was worse, averaging 7.9 months, whereas patients without leptomeningeal spread had an average overall survival of 12.2 months $(p=0.09)$. Of the four cases which had spread to the frontal lobe at autopsy, three were GBM (two K27M-H3 and one WT-H3) and one was AA (WT-H3.3).

\section{b}
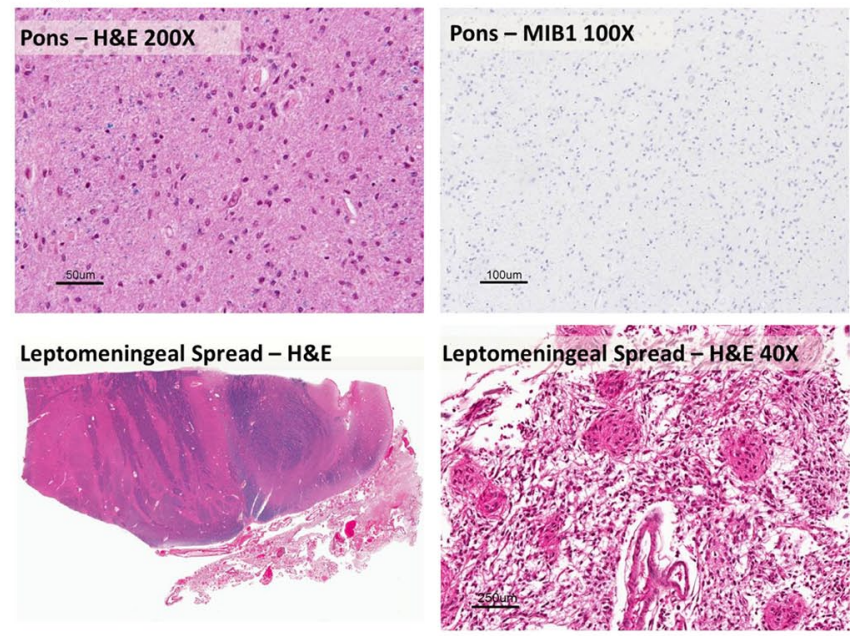

d
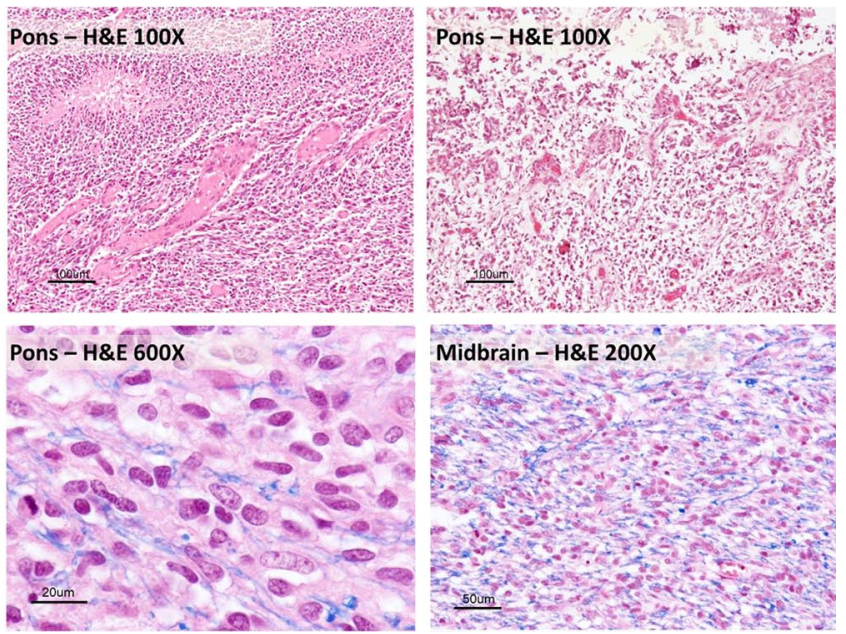

There was leptomeningeal spread of the tumour and an exophytic component involving both the pons and the medulla (lower panels). c GBM, K27M-H3.3: at autopsy this tumour has classic GBM features throughout the pons (upper left and right panels). There was diffuse intraparenchymal tumour infiltration into the thalamus (lower left panel) and cervical cord (lower right panel). d GBM, WT-H3.3: on autopsy, H\&E staining revealed histology consistent with that of GBM; including pseudopalisading necrosis (upper left panel) and vascular endothelial proliferation (upper right panel). There was diffuse intraparenchymal infiltration of the tumour into the midbrain (lower right panel)

Interestingly, the mean survival for the K27M-H3 mutant cases was 0.63 years and for the wild-type cases was 1.84 years, although the numbers are too small to make definitive conclusions based on this. Similarly, the small number of patients with this distant spread at autopsy did not allow for statistical analysis of tumour grade and H3-mutational status. Histone mutation status was available for 15 patients with leptomeningeal dissemination, of which $8(53 \%)$ had the K27M-H3.3 mutation. Gains of PDGFRA were detected in 4/8 DIPGs with K27M-H3.3 
Table 1 WHO diagnosis of diffuse intrinsic pontine glioma by tumour location
$G B M$ glioblastoma grade IV, $A A$ anaplastic astrocytoma grade III, $D A$ diffuse astrocytoma grade II

\begin{tabular}{lllllll}
\hline Autopsy cases & Pons & Medulla & Midbrain & Thalamus & Frontal lobe & Mets \\
\hline DIPG03 & GBM & DA & AA & DA & - & - \\
DIPG04 & GBM & AA & AA & DA & - & - \\
DIPG05 & GBM & GBM & AA & AA & - & GBM \\
DIPG06 & GBM & DA & GBM & - & - & GBM \\
DIPG07 & GBM & DA & GBM & AA & - & - \\
DIPG08 & GBM & AA & GBM & - & - & - \\
DIPG18 & GBM & AA & AA & AA & - & - \\
DIPG19 & AA & AA & AA & AA & - & GBM \\
DIPG24 & GBM & AA & AA & - & - & - \\
DIPG27 & GBM & AA & GBM & AA & - & - \\
DIPG58 & GBM & AA & GBM & GBM & DA & - \\
DIPG59 & GBM & GBM & GBM & GBM & AA & - \\
DIPG60 & GBM & DA & AA & - & - & - \\
DIPG61 & GBM & AA & AA & AA & - & - \\
DIPG62 & GBM & AA & AA & DA & - & - \\
DIPG66 & GBM & AA & GBM & GBM & AA & - \\
DIPG78 & GBM & AA & GBM & - & - & - \\
\hline
\end{tabular}

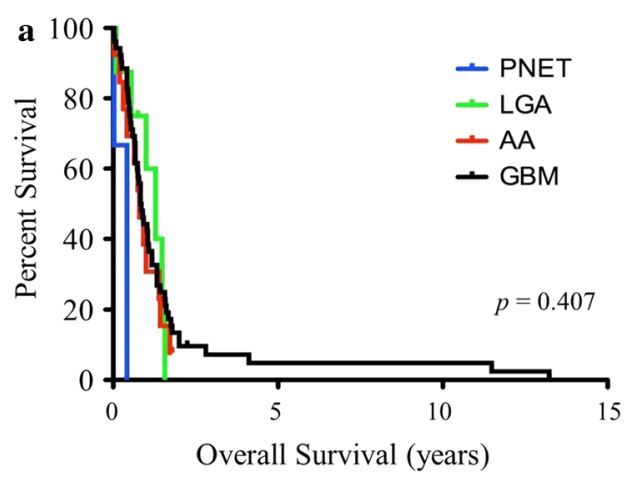

Fig. 2 Histone mutations but not histologic grade predicts survival of DIPG patients. a Kaplan-Meier analysis of DIPG based on tumour grade and histology reveals there is no significant difference in survival based on histologic diagnosis $(p=0.407)$. b DIPG patients with

mutation and leptomeningeal dissemination. TP53 mutation status was available for 11 patients with leptomeningeal disease. Six of these patients had TP53 mutations. One DIPG patient with leptomeningeal disease (GBM histology) had a p.Gly328Val substitution in ACVR1 and one patient (LGA histology) had a p.Glu545Gly alteration in PIK3CA. There was no statistical difference in the frequency of PIK3CA mutations between disseminated and non-disseminated cases.

\section{K27M-H3.3 mutations and histology}

Tumour tissue from the pons of 66 DIPG patients was screened for $\mathrm{K} 27 \mathrm{M}$ mutation in histone $\mathrm{H} 3$ as previously described [10]. 42/66 (64\%) were found to be mutated for

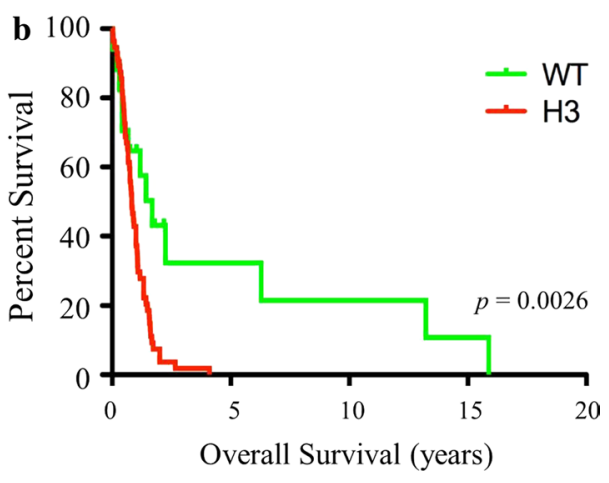

K27M-H3 mutation (red) have worse overall survival as compared to wild-type H3 DIPG patients (green) as determined by Kaplan-Meier analysis ( $\log$ rank, $p=0.0026)$

K27M-H3.3, with an additional eight patients with K27MH3.1 mutations $(12 \%)$. Mutant allele frequencies from deep sequencing suggest histone mutations are heterozygously present in all tumour cells. Of the 14 biopsy tested for histone mutations, 7 (50 \%) were mutated for K27MH3.3 and none had histone H3.1 mutations. Of the 52 autopsy cases tested for histone $\mathrm{H} 3$ mutations, 35 (67\%) were mutated for K27M-H3.3 and $8(15 \%)$ were mutated for K27M-H3.1. We previously performed survival analysis in a subset of these DIPG patients based on histone mutation status [10]. In this larger series, the observation that patients with K27M mutation in either histone H3.1 or H3.3 have worse overall survival compared to patients with no histone mutations remains significant (Fig. 2b, Log rank, $p=0.0026$ ). On multivariate analysis (Cox 
Table 2 Multivariate Cox regression analysis

\begin{tabular}{lllll}
\hline Variables & HR & $p$ value & Lower 95 \% CI & Upper 95 \% CI \\
\hline K27M-H3 & 2.793 & 0.006 & 1.350 & 5.777 \\
Histology & 0.808 & 0.222 & 0.574 & 1.138 \\
Age Dx & 1.016 & 0.693 & 0.940 & 1.097 \\
Sex & 1.267 & 0.412 & 0.720 & 2.231 \\
\hline
\end{tabular}

Age Dx age of diagnosis, $H R$ hazard ratio, $C I$ confidence interval

regression), which included histone mutational status, age of diagnosis, histological grade and sex, only histone mutation status was determined to be a significant predictor of overall survival with a hazard ratio of 2.8 (95\% confidence intervals, $1.35-5.78, p=0.006$, Table 2). DIPG patients who had wild-type $\mathrm{H} 3$ were diagnosed at a significantly younger age than patients with K27M-H3 (wildtype 4.84 years \pm 4.13 vs. K27M-H3 7.36 years \pm 3.31 , $p=0.018)$. There was also correlation between histologic grade and mutational status. Eighty-eight percent of GBMs harboured a K27M mutation in $\mathrm{H} 3$ versus $60 \%$ of AAs and $71 \%$ of LGA. K27M-H3.3 was found at a statistically higher ratio in GBM patients (78 \%) than patients with AA histology $(33 \%)(p=0.0016)$. The association of anaplastic astrocytoma histology and K27M-H3.1 mutations approached significance $(p=0.058)$. All K27M mutant tumours were glial (tumours with PNET histology were wild-type), but represented a spectrum of WHO grades (II-IV). There was no correlation between K27M-mutation status and leptomeningeal spread. Some patients were wild-type for H3.3 yet exhibited features of high-grade astrocytic tumours such as pseudopalisading necrosis, vascular endothelial proliferation and mitoses (Fig. 1d). Interestingly, despite typical high-grade glioma features, patients who were wild-type for H3 with GBM histology survived significantly longer than patients mutated for K27M with GBM histology (mean survival of 1.99 years vs. 0.96 years; $p=0.026$ ). Conversely, some DIPG patients exhibited low-grade tumours with low proliferative index, yet were mutated for K27M-H3.3 (Fig. 1b) and exhibited a clinical course typical of high-grade gliomas. DIPG with grade II and III histology carrying the mutation do just as poorly as GBM. The overall survival of K27M-H3.3 tumours with grade II and III histology was $0.82 \pm 0.47$ years compared to $0.91 \pm 0.77$ years for K27M-H3.3 grade IV tumours $(p>0.05)$. Patients with WHO grade II diffuse astrocytomas, but wild-type for $\mathrm{H} 3$ survived longer than K27M-H3.3 mutant WHO grade II astrocytomas. Histologically, these cases all had similar features of diffusely infiltrating astrocytic tumour with rare to no mitotic figures, low MIB1, diffuse GFAP immunopositivity and rare to no P53 immunopositivity. Most DIPGs were positive for GFAP, with more diffuse staining present in the grade II lesions and no GFAP immunopositivity present in DIPG with PNET histology.

DIPG histologic subgroups have unique genetic signatures

DIPG patients, although not significantly differing in their overall survival when based on histology and tumour grade, do show clinical and genetic alterations associated with histology. Sex differences were noted between the different histologies with GBM patients having a 2:1 male to female ratio. This trend was opposite in anaplastic astrocytomas. Mutations and copy number alterations in genes known to be associated with DIPG were also different among histologic subgroups. DIPG with GBM and AA histology had 65 and $25 \%$ incidence of TP53 mutations, respectively, while no TP53 mutations were found in lowgrade astrocytoma DIPG patient tumours. Immunostaining for P53 did not correlate with TP53 mutation status, possibly a result of unreliable results from post-mortem tissue. In addition, $50 \%$ of DIPG with both GBM and AA histology had deletion of one copy of TP53. As with mutations, no grade II DIPG had this alteration. A similar association was seen with activating mutations of ACVRl, where 20 and $25 \%$ of patients with GBM and AA histology had these mutations, respectively, whereas grade II DIPG and DIPG with PNET histology did not harbour ACVRI mutations. PIK3CA mutations were present in $14.3 \%$ of DIPGs, including WHO grade II-IV astrocytomas but not PNETs. Similarly, PDGFRA copy number gains were seen in between 36 and $43 \%$ of patients among all astrocytic histologies of DIPG and not in any PNET. Alternate lengthening of telomeres (ALT) phenotype was seen in 28 and $17 \%$ of GBM and AA patients but not in LGA or PNET DIPG patients (Fig. 3).

\section{Discussion}

Our results highlight the clinicopathological heterogeneity of DIPG and describe the relationship between histology, leptomeningeal dissemination clinical outcome and molecular features. Based on WHO guidelines, DIPG have histology corresponding to grade II, grade III and grade IV tumours. Several cases with typical MRI and symptoms at presentation had histologies on autopsy that would not be considered "classic" for DIPG such as PNET or LGA. Previous case studies have reported DIPG with PNET histology [23]. As in our cases, they described these tumours as having poorly differentiated cells, with scant cytoplasm and round nuclei. Interestingly, on gene expression profiling, these tumours resembled supratentorial PNET [23]. Patients in our series who were found at autopsy to have PNET were diagnosed as DIPG based on standard criteria 


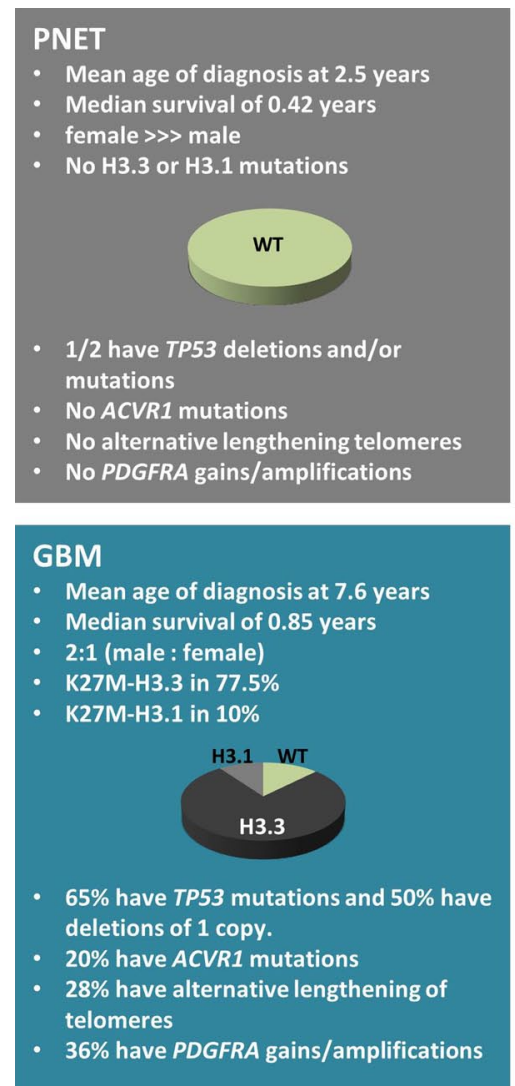

\section{DIPG Histologic Subgroups}

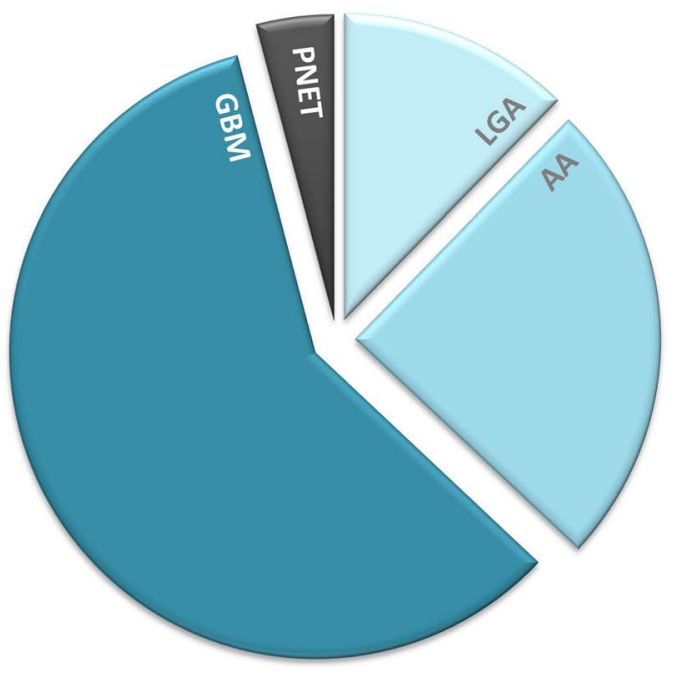

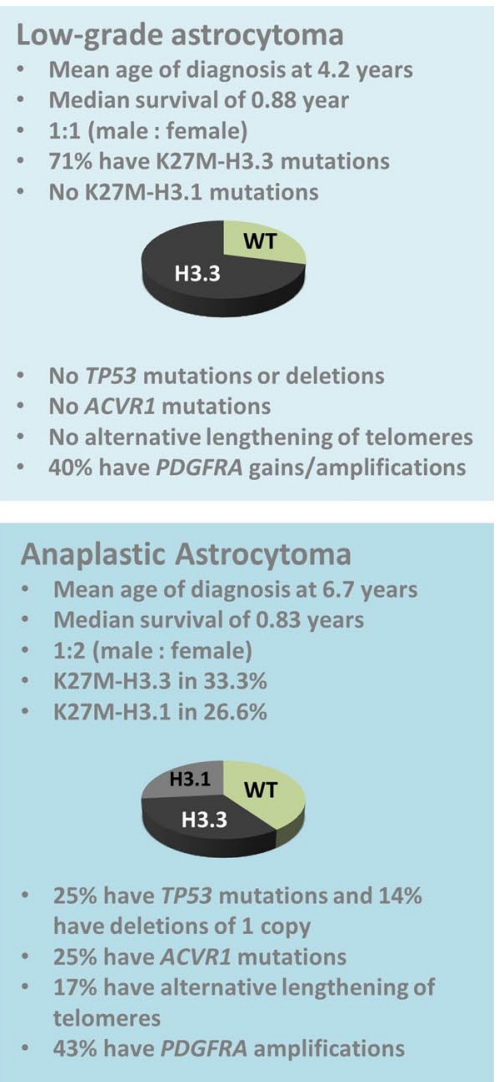

Fig. 3 DIPG histologic subgroups have some unique clinical and molecular features including mean age of diagnosis, sex, H3.3 and H3.1 mutations, TP53 mutations and deletions, ACVR1 mutations, PDGFRA gains/amplifications and alternative lengthening of telomeres (ALT)

including MRI and clinical presentation. These patients had clinical course of what is expected in the spectrum for "classical" DIPG and while an argument can be made that PNET tumours of the brainstem should not be included as pontine gliomas, the purpose of our study was to investigate the molecular and histologic spectrum of what is clinically called DIPG. If biopsy becomes standard and diagnosis incorporates histologic and molecular criteria, these entities may be given an alternate designation.

Although there is a wide distribution of tumour grade and histology among DIPG, these alone are not a predictor of survival. A previous clinicopathological study by Yoshimura et al. [25] of 40 diffuse brainstem tumours (33 paediatric and 7 adult) reported a significant difference in overall survival between GBM and AA patients. This difference may be the result of inclusion of adult patients in this cohort. When considering only the paediatric population of the previous study, only four patients with AA and one patient with LGA histology were described and the K27M-H3.3 status, which defines clinically relevant subgroups of DIPG [10], was not known. We found some association between histologic grade (GBM grade IV) and H3.3 mutational status, however, K27M-mutated tumours are found in DIPG with grade II, III and IV astrocytoma histology (Fig. 3). Tumours with lower-grade histology carrying the $\mathrm{H} 3.3$ mutation had overall survival comparable to that of grade IV GBM tumours. While patients with highgrade tumours wild-type for $\mathrm{H} 3.3$ had overall survival comparable to patients with wild-type low-grade astrocytomas. K27M mutation testing may be helpful for biopsy specimen diagnosis, particularly given the potential histologic intratumoural heterogeneity observed in DIPG, as some tumours with classic GBM histology possess grade II or grade III histology areas, which may be targeted on biopsy. A majority of these heterogeneous cases had features of classic GBM in the pons, including vascular endothelial proliferation and necrosis; however, when sampling the midbrain or thalamus, these features were not present and histology was consistent with LGA or AA. While wild-type status does not imply a better survival (for example, the PNET-like tumours were wild-type for H3.3 and had poor outcome), the presence of a $\mathrm{K} 27-\mathrm{H} 3$ mutation is associated with poor outcome, despite histologic appearance. With advent of biopsy-driven clinical trials [12], K27M mutational status may be pivotal in trial design, particularly when a tumour is classified as a grade II due to limited biopsy specimen. 
Distribution of PDGFRA gains/amplification as well as PIK3CA mutations was similar among all astrocytic tumours, irrespective of grade, whereas ALT phenotype and mutations in TP53 and ACVRI were only observed in tumours with AA and GBM histologies. None of the $P D G$ FRA gains/amplifications, PIK3CA mutations, ALT phenotype or ACVRI mutations were found in tumours with PNET histology.

Thirty-eight percent of patients exhibited leptomeningeal spread of their tumour. The prevalence of leptomeningeal dissemination and distant tumour spread in our series of patients was similar to that reported previously [25]. We found no association with leptomeningeal dissemination and histology. The frequency of certain molecular features common amongst DIPG such as histone $\mathrm{H} 3$ mutations, PDGFRA gains/amplifications, ACVRI and PIK3CA mutations were not enriched in patients with leptomeningeal dissemination. Interestingly, none of the patients with leptomeningeal dissemination were found to have ALT phenotype. Diffuse tumour invasion of the brainstem was common among all of the DIPGs; with $25 \%$ diffusely involving the upper cervical cord and thalamus. Some patients exhibited distal spread as far as the frontal lobes. This observation may be critical for clinical decisionmaking, as focal radiation may be inadequate for patients with leptomeningeal and/or extra-brainstem spread. These patients may benefit from new treatment strategies targeted towards inhibiting tumour infiltration rather than proliferation, especially since some of these very infiltrative tumours exhibit few mitoses and scarce MIB1 positivity. Conversely, on autopsy two patients had severe radiation necrosis of the brainstem with several other patients showing mild to moderate radiation necrosis.

Based on the histologic spectrum of DIPG as well as the prognostic and therapeutic implications of K27M-H3, our findings argue for testing H3-mutation status of biopsy specimens at diagnosis. This important genetic information could be rapidly integrated into the clinical practice, particularly in atypical DIPG, such as those with PNET histology or low-grade lesions with mutant histone H3 that have aggressive clinical course not typically encountered in supratentorial LGA. Importantly, the WHO grading scheme does not predict outcome in DIPG patients. New therapeutic approaches need to incorporate molecular and histological data in order to achieve maximum benefit for DIPG patients.

Acknowledgments We would like to thank Dr. Anne Bendel (Children's Hospital Minneapolis), Drs. Jennifer Chang and Lucie LafayCousin (University of Calgary), Drs. Sandra Dunn, Juliette Hukin and Chris Dunham (BC Children's Hospital), Dr. Katrin Scheinemann (McMaster Children's Hospital), Dr. Jean Mchaud (Children's Hospital of Eastern Ontario), Drs. Shayna Zelcer and David Ramsay (London Health Sciences Centre), Dr. Javad Nazarian (George
Washington University), Dr. Jason Fangusaro (Children's Hospital of Chicago), Dr. Matthias Karajannis (NYU Langone Medical Center), Dr. Nicholas Foreman (University of Colorado), Drs. Julia Hegert and Amy Smith (Arnold Palmer Hospital for Children), Dr. Mark Souweidane (Weill Cornell Medical College) and Dr. Jason Cain (Monash Institute of Cancer Research) for providing us with DIPG biological material for this study. This work was supported by an operating grant from the National Brain Tumor Society and the Canadian Institutes of Health Research [MOP 115004]. Pawel Buczkowicz is a recipient of CIHR Doctoral Frederick Banting and Charles Best Canada Graduate Scholarships award.

Open Access This article is distributed under the terms of the Creative Commons Attribution License which permits any use, distribution, and reproduction in any medium, provided the original author(s) and the source are credited.

\section{References}

1. Bender S, Tang Y, Lindroth AM, Hovestadt V, Jones DT, Kool M, Zapatka M, Northcott PA, Sturm D, Wang W, Radlwimmer B, Hojfeldt JW, Truffaux N, Castel D, Schubert S, Ryzhova M, Seker-Cin H, Gronych J, Johann PD, Stark S, Meyer J, Milde T, Schuhmann M, Ebinger M, Monoranu CM, Ponnuswami A, Chen S, Jones C, Witt O, Collins VP, von Deimling A, Jabado N, Puget S, Grill J, Helin K, Korshunov A, Lichter P, Monje M, Plass C, Cho YJ, Pfister SM (2013) Reduced H3K27me3 and DNA hypomethylation are major drivers of gene expression in K27M mutant pediatric high-grade gliomas. Cancer Cell 24(5):660-672. doi:10.1016/j.ccr.2013.10.006

2. Bernstein BE, Mikkelsen TS, Xie X, Kamal M, Huebert DJ, Cuff J, Fry B, Meissner A, Wernig M, Plath K, Jaenisch R, Wagschal A, Feil R, Schreiber SL, Lander ES (2006) A bivalent chromatin structure marks key developmental genes in embryonic stem cells. Cell 125(2):315-326. doi:10.1016/j.cell.2006.02.041

3. Buczkowicz P, Hoeman C, Rakopoulos P, Pajovic S, Letourneau L, Dzamba M, Morrison A, Lewis P, Bouffet E, Bartels U, Zuccaro J, Agnihotri S, Ryall S, Barszczyk M, Chornenkyy Y, Bourgey M, Bourque G, Montpetit A, Cordero F, Castelo-Branco P, Mangerel J, Tabori U, Ho KC, Huang A, Taylor KR, Mackay A, Bendel AE, Nazarian J, Fangusaro JR, Karajannis MA, Zagzag D, Foreman NK, Donson A, Hegert JV, Smith A, Chan J, Lafay-Cousin L, Dunn S, Hukin J, Dunham C, Scheinemann K, Michaud J, Zelcer S, Ramsay D, Cain J, Brennan C, Souweidane MM, Jones C, Allis CD, Brudno M, Becher O, Hawkins C (2014) Genomic analysis of diffuse intrinsic pontine gliomas identifies three molecular subgroups and recurrent activating ACVR1 mutations. Nat Genet 46(5):451-456. doi:10.1038/ng.2936

4. Freeman CR, Perilongo G (1999) Chemotherapy for brain stem gliomas. Childs Nerv Syst 15(10):545-553

5. Grill J, Puget S, Andreiuolo F, Philippe C, MacConaill L, Kieran MW (2012) Critical oncogenic mutations in newly diagnosed pediatric diffuse intrinsic pontine glioma. Pediatr Blood Cancer 58(4):489-491. doi:10.1002/pbc. 24060

6. Hargrave D, Bartels U, Bouffet E (2006) Diffuse brainstem glioma in children: critical review of clinical trials. Lancet Oncol 7(3):241-248

7. Henson JD, Cao Y, Huschtscha LI, Chang AC, Au AY, Pickett HA, Reddel RR (2009) DNA C-circles are specific and quantifiable markers of alternative-lengthening-of-telomeres activity. Nat Biotechnol 27(12):1181-1185. doi:10.1038/nbt.1587

8. Herbert BS, Shay JW, Wright WE (2003) Analysis of telomeres and telomerase. Current protocols in cell biology/editorial board, 
Juan S Bonifacino et al. Chapter 18: Unit 18 16. doi:10.1002/047 1143030.cb1806s20

9. Jansen MH, van Vuurden DG, Vandertop WP, Kaspers GJ (2012) Diffuse intrinsic pontine gliomas: a systematic update on clinical trials and biology. Cancer Treat Rev 38(1):27-35. doi:10.1016/j.ctrv.2011.06.007

10. Khuong-Quang DA, Buczkowicz P, Rakopoulos P, Liu XY, Fontebasso AM, Bouffet E, Bartels U, Albrecht S, Schwartzentruber J, Letourneau L, Bourgey M, Bourque G, Montpetit A, Bourret G, Lepage P, Fleming A, Lichter P, Kool M, von Deimling A, Sturm D, Korshunov A, Faury D, Jones DT, Majewski J, Pfister SM, Jabado N, Hawkins C (2012) K27M mutation in histone H3.3 defines clinically and biologically distinct subgroups of pediatric diffuse intrinsic pontine gliomas. Acta Neuropathol 124(3):439-447. doi:10.1007/s00401-012-0998-0

11. Lewis PW, Muller MM, Koletsky MS, Cordero F, Lin S, Banaszynski LA, Garcia BA, Muir TW, Becher OJ, Allis CD (2013) Inhibition of PRC2 activity by a gain-of-function $\mathrm{H} 3$ mutation found in pediatric glioblastoma. Science 340(6134):857-861. doi:10.1126/science.1232245

12. MacDonald TJ (2012) Diffuse intrinsic pontine glioma (DIPG): time to biopsy again? Pediatr Blood Cancer 58(4):487-488. doi:10.1002/pbc. 24090

13. Maria BL, Rehder K, Eskin TA, Hamed LM, Fennell EB, Quisling RG, Mickle JP, Marcus RB Jr, Drane WE, Mendenhall NP et al (1993) Brainstem glioma: I. Pathology, clinical features, and therapy. J Child Neurol 8(2):112-128

14. Meissner A, Mikkelsen TS, Gu H, Wernig M, Hanna J, Sivachenko A, Zhang X, Bernstein BE, Nusbaum C, Jaffe DB, Gnirke A, Jaenisch R, Lander ES (2008) Genome-scale DNA methylation maps of pluripotent and differentiated cells. Nature 454(7205):766-770. doi:10.1038/nature07107

15. Messner S, Altmeyer M, Zhao H, Pozivil A, Roschitzki B, Gehrig P, Rutishauser D, Huang D, Caflisch A, Hottiger MO (2010) PARP1 ADP-ribosylates lysine residues of the core histone tails. Nucleic Acids Res 38(19):6350-6362. doi:10.1093/nar/gkq463

16. Paugh BS, Broniscer A, Qu C, Miller CP, Zhang J, Tatevossian RG, Olson JM, Geyer JR, Chi SN, da Silva NS, Onar-Thomas A, Baker JN, Gajjar A, Ellison DW, Baker SJ (2011) Genome-wide analyses identify recurrent amplifications of receptor tyrosine kinases and cell-cycle regulatory genes in diffuse intrinsic pontine glioma. Journal of clinical oncology Official journal of the American Society of Clinical Oncology 29(30):3999-4006

17. Paugh BS, Qu C, Jones C, Liu Z, Adamowicz-Brice M, Zhang J, Bax DA, Coyle B, Barrow J, Hargrave D, Lowe J, Gajjar A, Zhao W, Broniscer A, Ellison DW, Grundy RG, Baker SJ (2010) Integrated molecular genetic profiling of pediatric high-grade gliomas reveals key differences with the adult disease. J Clin Oncol 28(18):3061-3068. doi:10.1200/JCO.2009.26.7252

18. Puget S, Philippe C, Bax DA, Job B, Varlet P, Junier MP, Andreiuolo F, Carvalho D, Reis R, Guerrini-Rousseau L, Roujeau T, Dessen P, Richon C, Lazar V, Le Teuff G, Sainte-Rose C, Geoerger B, Vassal G, Jones C, Grill J (2012) Mesenchymal transition and PDGFRA amplification/mutation are key distinct oncogenic events in pediatric diffuse intrinsic pontine gliomas. PLoS One 7(2):e30313. doi:10.1371/journal.pone.0030313

19. Ramos A, Hilario A, Lagares A, Salvador E, Perez-Nunez A, Sepulveda J (2013) Brainstem gliomas. Semin Ultrasound CT MR 34(2):104-112. doi:10.1053/j.sult.2013.01.001

20. Reynolds N, Salmon-Divon M, Dvinge H, Hynes-Allen A, Balasooriya G, Leaford D, Behrens A, Bertone P, Hendrich B (2012) NuRD-mediated deacetylation of $\mathrm{H} 3 \mathrm{~K} 27$ facilitates recruitment of polycomb repressive complex 2 to direct gene repression. EMBO J 31(3):593-605. doi:10.1038/emboj.2011.431

21. Robison NJ, Kieran MW (2014) Diffuse intrinsic pontine glioma: a reassessment. J Neurooncol. doi:10.1007/s11060-014-1448-8

22. Schwartzentruber J, Korshunov A, Liu XY, Jones DT, Pfaff E, Jacob K, Sturm D, Fontebasso AM, Quang DA, Tonjes M, Hovestadt V, Albrecht S, Kool M, Nantel A, Konermann C, Lindroth A, Jager N, Rausch T, Ryzhova M, Korbel JO, Hielscher T, Hauser P, Garami M, Klekner A, Bognar L, Ebinger M, Schuhmann MU, Scheurlen W, Pekrun A, Fruhwald MC, Roggendorf W, Kramm C, Durken M, Atkinson J, Lepage P, Montpetit A, Zakrzewska M, Zakrzewski K, Liberski PP, Dong Z, Siegel P, Kulozik AE, Zapatka M, Guha A, Malkin D, Felsberg J, Reifenberger $\mathrm{G}$, von Deimling A, Ichimura K, Collins VP, Witt H, Milde T, Witt O, Zhang C, Castelo-Branco P, Lichter P, Faury D, Tabori U, Plass C, Majewski J, Pfister SM, Jabado N (2012) Driver mutations in histone $\mathrm{H} 3.3$ and chromatin remodelling genes in paediatric glioblastoma. Nature 482(7384):226-231. doi:10.1038/nature10833

23. Sufit A, Donson AM, Birks DK, Knipstein JA, Fenton LZ, Jedlicka P, Hankinson TC, Handler MH, Foreman NK (2012) Diffuse intrinsic pontine tumors: a study of primitive neuroectodermal tumors versus the more common diffuse intrinsic pontine gliomas. J neurosurg Pediatr 10(2):81-88. doi:10.3171/2012.3.P EDS11316

24. Wu G, Broniscer A, McEachron TA, Lu C, Paugh BS, Becksfort J, Qu C, Ding L, Huether R, Parker M, Zhang J, Gajjar A, Dyer MA, Mullighan CG, Gilbertson RJ, Mardis ER, Wilson RK, Downing JR, Ellison DW, Zhang J, Baker SJ, St. Jude Children's Research Hospital-Washington University Pediatric Cancer Genome P (2012) Somatic histone H3 alterations in pediatric diffuse intrinsic pontine gliomas and non-brainstem glioblastomas. Nature genet 44(3):251-253. doi:10.1038/ng.1102

25. Yoshimura J, Onda K, Tanaka R, Takahashi H (2003) Clinicopathological study of diffuse type brainstem gliomas: analysis of 40 autopsy cases. Neurologia medico-chirurgica 43(8):375-382 (discussion 382)

26. Zarghooni M, Bartels U, Lee E, Buczkowicz P, Morrison A, Huang A, Bouffet E, Hawkins C (2010) Whole-genome profiling of pediatric diffuse intrinsic pontine gliomas highlights plateletderived growth factor receptor alpha and poly (ADP-ribose) polymerase as potential therapeutic targets. J clin oncol 28(8):13371344. doi:10.1200/JCO.2009.25.5463 